\title{
On the equivalence of Reggeon field theory in zero transverse dimensions and reaction-diffusion processes
}

\author{
S. Bondarenko $^{a) *}$, L. Motyka ${ }^{b), c) \dagger}$, A.H. Mueller ${ }^{d) \ddagger}$, A.I. Shoshi ${ }^{e)} \S$ and B.-W. Xiao ${ }^{d)} \uparrow$ \\ a) II Institute for Theoretical Physics, University of Hamburg, 22761 Hamburg, Germany \\ b) DESY Theory Group, 22603 Hamburg, Germany \\ c) Institute of Physics, Jagellonian University, 30-059 Kraków, Poland \\ d) Physics Department, Columbia University, New York, NY-10027, USA \\ e) Fakultät für Physik, Universität Bielefeld, D-33501 Bielefeld, Germany
}

September 20, 2006

\begin{abstract}
The Reggeon field theory in zero transverse dimensions is investigated. Two versions of the theory are considered: one that allows at most triple pomeron interactions and the other that embodies an additional $2 \rightarrow 2$ quartic Reggeon coupling. The behavior of the scattering amplitude at asymptotic rapidities is obtained in both cases. In an $s$-channel picture of the high energy scattering both models can be viewed as reaction-diffusion processes. We derive known results in Reggeon field theory rather easily using the reaction-diffusion formalism. We find that some results which are surprising from the Reggeon field theory point of view turn out to have a simple interpretation from the reaction-diffusion point of view.
\end{abstract}

\footnotetext{
*Email: sergb@mail.desy.de

${ }^{\dagger}$ E-mail: motyka@th.if.uj.edu.pl

${ }^{\ddagger}$ E-mail: arb@phys.columbia.edu

$\S$ E-mail: shoshi@physik.uni-bielefeld.de

『E-mail: bowen@phys.columbia.edu
} 


\section{Introduction}

The high energy scattering in QCD may be described in terms of a non-local field theory of QCD pomerons. In this formulation the basic degrees of freedom — the BFKL pomerons [1, 2, 3, 4, — merge and split according to non-local multi-pomeron vertices [5, 6, 7, 8, 9, 10, 11, 12, 13]. This approximation is consistent with the unitarity of the $S$-matrix. Alternative approaches to the unitarization of high energy amplitudes use extensively methods of statistical physics combined with field-theoretical calculations in QCD, see e.g. Refs. [14] 34. The coordinate space in all those formulations is the transverse plane to the axis of the high energy collision. In all cases the emerging evolution equations for high energy amplitudes are non-local functional equations which are, so far, prohibitively difficult to solve exactly. Therefore, it is useful to study a substantially simplified model of the interacting pomeron theory, in which the dependence of the pomeron fields on transverse coordinates is discarded. The emerging simplified model - Reggeon field theory (RFT) in zero transverse dimensions - was proposed and formally solved long time ago [35, 36, 37, 38, 39, 40. This toy model exhibits some features which are present also in the more realistic QCD pomeron field theory. Therefore, RFT in zero transverse dimensions offers a useful testing ground for computational methods and recently it enjoys revived interest [41, 42, 43, 44, 45, 46]. In this paper we will explore the equivalence between RFT and the statistical reaction-diffusion picture of high energy scattering that holds in zero transverse dimensions.

In Section 2 we consider the zero dimensional Reggeon field theory. Equations of RFT are derived from the Langevin formulation and from the Lagrangian of RFT. Two distinct realizations of RFT are studied: a minimal RFT with triple pomeron vertices only (which will be abbreviated as MRFT) and a theory with an additional $2 \rightarrow 2$ pomeron interaction which is equivalent to a simple reaction-diffusion $s$-channel model (which will be called the reaction-diffusion RFT or RD-RFT). Asymptotic behavior of scattering amplitudes in both RFTs is obtained. The minimal RFT yields a counter-intuitive exponential decrease for the scattering amplitude $T(Y) \sim \exp \left(-E_{0} Y\right)$ at very large rapidities $Y$, with the leading order result of $E_{0}$ given by $E_{0} \sim \exp \left(-\mu^{2} / 2 \lambda^{2}\right)$ where $\mu$ denotes the pomeron intercept and $\lambda$ the triple pomeron coupling. Clearly, the dependence of $E_{0}$ on the triple pomeron coupling is non-perturbative.

The presence of a quartic $2 \rightarrow 2$ pomeron coupling, $\lambda^{\prime}$, changes this picture. Namely, since $E_{0}=0$

for $\lambda^{\prime}=\lambda^{2} / \mu$, the scattering amplitude approaches a constant value at asymptotic rapidities instead of going to zero. In this case, the exact asymptotic solution of the RFT is given. Furthermore, we briefly discuss two possible options of multi-pomeron couplings to elementary external sources. Firstly, the eikonal couplings that were usually assumed in RFT. In the other scenario only a single pomeron coupling to the elementary external source is allowed. The latter scheme is realized in QCD where (at the leading logarithmic approximation) only single BFKL pomeron couples to an elementary colour dipole [6, 7, 8, 9]. It is also favored by the $s$-channel reaction-diffusion picture in which each 
elementary particle in the cascade may couple to at most one $t$-channel exchange. In this context, it is particularly interesting to consider the RFT counterpart of the reaction-diffusion model in which only single coupling to external sources are allowed with the strength $\alpha \ll 1$ related to the pomeron intercept $\mu=\alpha$ and the triple pomeron coupling $\lambda=\alpha^{2}$. In this model the high energy asymptotics, $Y \rightarrow \infty$, of the scattering amplitude happens to be close to the unitarity limit:

$$
\tilde{T}(Y) \longrightarrow \frac{1}{1-e^{-1 / \alpha^{2}}},
$$

which should be contrasted with the result of RD-RFT with the same parameters and eikonal couplings:

$$
T(Y) \longrightarrow \frac{(1-1 / e)^{2}}{1-e^{-1 / \alpha^{2}}}
$$

In Section 3 we give a complementary analysis of the scattering amplitudes in zero transverse dimensions in the probabilistic reaction-diffusion framework. It turns out, that the minimal RFT may be represented in the $s$-channel picture, analogously to the reaction-diffusion process. The transition rates, however, do not obey the constraints of the Markovian process - the transition coefficients are negative that prohibits the genuine probabilistic interpretation. Nevertheless, the quasi-reactiondiffusion picture provides an efficient calculational framework. Thus, we obtain results for high rapidity asymptotics of scattering amplitudes that coincide with ones from the conventional RFT framework, but the interpretation is more transparent. We also demonstrate the equivalence of the results obtained within the proper reaction-diffusion framework with the results of the corresponding RFT. Finally, the representation of the eikonal model in the reaction-diffusion framework is given. Curiously, the "grey disc" limit of the scattering amplitudes given by Eq. (1.2) emerges as a consequence of the normalisation of the scattering states that is smaller than one.

Finally, we note that a different $s$-channel picture has recently been elaborated in Ref. [46] which is very different than the one we have here discussed. The eikonal couplings are used and boost invariance is preserved by having arbitrarily high Reggeon vertices in the hierarchy equations. The system does not reach a fixed point, but rather reaches a steady rate of increase of particles with increasing rapidity. It will be interesting to try and further clarify which of these pictures, if either, has the closest relationship with four-dimensional QCD.

\section{Reggeon field theory in zero transverse dimensions}

\subsection{Reggeon field theory from the Langevin equation.}

The general form of the Langevin equation in the Ito formulation is

$$
d u(y+d y)=D_{1}(u(y)) d y+D_{2}(u(y)) d \omega(y)
$$


where $u(y)$ is a stochastic function of an evolution variable $y, d y$ is an infinitesimally small step of $y$, and $\omega(y)$ is related to a random noise variable $\nu(y)$,

$$
d \omega(y)=\nu(y) d y
$$

The noise is taken to be Gaussian,

$$
\langle\nu(y)\rangle=0, \quad\left\langle\nu(y) \nu\left(y^{\prime}\right)\right\rangle=\delta\left(y-y^{\prime}\right) .
$$

Let us consider a dynamical quantity $G(u)$ with $u$ distributed with the probability density $p(y, u)$. It follows from the Ito calculus that the average value of this variable

$$
\langle G\rangle_{y}=\int d u p(y, u) G(u)
$$

evolves according to the following master equation:

$$
\frac{d\langle G\rangle_{y}}{d y}=\left\langle D_{1}(u) \frac{d G}{d u}\right\rangle_{y}+\left\langle\frac{D_{2}^{2}(u)}{2} \frac{d^{2} G(u)}{d u^{2}}\right\rangle_{y} .
$$

Thus, assuming that moments of the variable $u$,

$$
\left\langle u^{k}\right\rangle_{y}=\int d u p(y, u) u^{k}
$$

obey the hierarchy of (deterministic) equations

$$
\frac{d\left\langle u^{k}\right\rangle_{y}}{d y}=k\left\langle D_{1}(u) u^{k-1}\right\rangle_{y}+k(k-1)\left\langle\frac{D_{2}^{2}(u)}{2} u^{k-2}\right\rangle_{y} .
$$

which closes if $D_{1}(u)$ and $D_{2}^{2}(u)$ are analytic functions of $u$. With an appropriate choice of the functions $D_{1}(u)$ and $D_{2}(u)$ this hierarchy of equations may be made equivalent to the equations of the Reggeon field theory in zero transverse dimensions. We choose the following form of $D_{1}(u)$ and $D_{2}(u)$

$$
\begin{aligned}
& D_{1}(u)=\alpha u-\beta u^{2}, \\
& D_{2}^{2}(u)=2\left(\alpha^{\prime} u-\beta^{\prime} u^{2}\right),
\end{aligned}
$$

which is equivalent to RFT with triple pomeron vertices and a quartic $2 \rightarrow 2$ pomeron vertex. Indeed, with (2.8) and (2.9), one obtains from (2.7) a closed set of equations

$$
\frac{d\left\langle u^{k}\right\rangle_{y}}{d y}=\alpha k\left\langle u^{k}\right\rangle_{y}+\alpha^{\prime} k(k-1)\left\langle u^{k-1}\right\rangle_{y}-\beta k\left\langle u^{k+1}\right\rangle_{y}-\beta^{\prime} k(k-1)\left\langle u^{k}\right\rangle_{y} .
$$

As it will be shown, those equations are the same as equations of RFT if the $k$-pomeron amplitude $\Phi_{k}(y)$ is connected to the $k$-th moment $\left\langle u^{k}\right\rangle_{y}$ in the following way,

$$
\Phi_{k}(y)=-(-\alpha)^{k} \frac{\left\langle u^{k}\right\rangle_{y}}{k !}
$$

and the parameters $\alpha^{\prime}, \beta$ and $\beta^{\prime}$ are suitably adjusted. 


\subsection{Hamiltonian formulation of RFT}

The analysis in this and the following parts of this section recapitulates the formulation and the key results that were obtained within RFT long ago [36, 37, 38, 39, 40]. We describe them in some details in order to facilitate a comparison with the following analysis within the $s$-channel framework.

Originally, RFT was formulated as a field theory (or as quantum mechanics in zero transverse dimensions) of pomerons. The basic degrees of freedom in this formulation are the Gribov fields $\psi$ and $\psi^{+}$that create and annihilate the pomeron. The action defining the theory with triple pomeron couplings only (MRFT) is defined in the following way:

$$
S=\int d y\left\{\psi^{+} \partial_{y} \psi-\mu \psi^{+} \psi+i \lambda \psi^{+}\left(\psi^{+}+\psi\right) \psi\right\}
$$

where $\mu$ is the bare intercept of the pomeron and $\lambda$ is the coupling of the triple pomeron interaction. Note that the action is symmetric under the transform

$$
\psi \longleftrightarrow \psi^{+}, \quad y \rightarrow-y,
$$

which corresponds to the symmetry between the target and the projectile. After redefinition of the Gribov fields, $q=i \psi^{+}$and $p=i \psi$ the action may be rewritten in terms of real quantities

$$
S=\int d y\left\{p \partial_{y} q+\mu q p-\lambda q(q+p) p\right\}
$$

Then, the Hamiltonian of the problem is given by

$$
H=\mu q p-\lambda q(q+p) p
$$

The commutation relation of the pomeron annihilation and creation operators, $\left[\psi, \psi^{+}\right]=1$, implies $[p, q]=-1$ which may be realized for instance by identification of $q$ with a position operator and $p$ with a differential operator,

$$
p=-\frac{\partial}{\partial q} .
$$

In this representation the Hamiltonian takes the form

$$
H=-\left(\mu q-\lambda q^{2}\right) \frac{\partial}{\partial q}-\lambda q \frac{\partial^{2}}{\partial q^{2}}
$$

In the following the ratio $\varrho=\mu / \lambda$ will be used, which is the large parameter of our model.

The interacting pomeron system whose state is given by $\Psi(y, q)$ evolves according to a Schrödinger equation (with an imaginary evolution variable)

$$
-\frac{\partial \Psi}{\partial y}=H \Psi
$$


The complete definition of the problem requires that the domain of $q$ and the boundary conditions are specified. This, however, depends on the particular choice of the couplings. For the minimal RFT it was shown that it is sufficient to consider $0 \leq q \leq \infty$ with $\Psi(y, q)$ that obeys the conditions

$$
\Psi(y, 0)=0, \quad \lim _{q \rightarrow \infty} \partial_{q} \Psi(y, q)=0
$$

at all rapidities $y$.

The state $\Psi(y, q)$ embodies the complete information about $k$-pomeron amplitudes $\Phi_{k}(y)$ in the $t$-channel,

$$
\Psi(y, q)=\sum_{k=1}^{\infty} \Phi_{k}(y) q^{k}
$$

The following hierarchy of equations, equivalent to (2.18),

$$
\frac{d \Phi_{k}}{d y}=\mu k \Phi_{k}+\lambda k(k+1) \Phi_{k+1}-\lambda(k-1) \Phi_{k-1}, \quad k=1,2, \ldots
$$

is identical to the hierarchy of equations (2.10) describing the evolution of moments $\left\langle u^{k}\right\rangle$ in the Langevin formulation after the substitution (2.11) provided that it is set

$$
\alpha=\mu, \quad \alpha \alpha^{\prime}=\beta / \alpha=\lambda, \quad \beta^{\prime}=0 .
$$

Note, that the target-projectile symmetry of RFT imposes the constraint

$$
\beta=\alpha^{2} \alpha^{\prime}
$$

on the Langevin formulation.

\subsection{External couplings and scattering amplitudes}

The scattering amplitudes in the Reggeon field theory may be obtained after the bulk action given by Eq. (2.12) is supplemented by the action describing the coupling of the pomeron system to external particles. In the original formulation of RFT the eikonal couplings were assumed, that is the coupling of $k$ pomerons to the external source was given by a product of $k$ single pomeron couplings multiplied by the combinatoric symmetry factor, $1 / k$ ! for $k$ outgoing pomerons ${ }^{1}$. In this scenario, the initial condition of the RFT Hamiltonian evolution is obtained by a resummation of multi-pomeron source amplitudes:

$$
\Psi(y=0, q)=\sum_{k=1}^{\infty} \Phi_{k}(0) q^{k}=\sum_{k=1}^{\infty} \frac{-\left(-g_{1}\right)^{k} q^{k}}{k !}=1-\exp \left(-g_{1} q\right),
$$

\footnotetext{
${ }^{1}$ This assumption seems to be rather natural for interacting point-like objects, but it may be not applicable in the case of QCD pomerons. In particular, due to a complex non-local nature of the BFKL pomeron, only one BFKL pomeron can couple to an elementary colour dipole in the leading logarithmic approximation.
} 
where $g_{1}$ is the coupling of the pomeron to the source. Thus, the scattering amplitude in RFT at rapidity $y$ is given by

$$
T\left(Y ; g_{1}, g_{2}\right)=\sum_{k=1}^{\infty} \Phi_{k}(Y) g_{2}^{k}=\Psi\left(Y, g_{2}\right),
$$

where $g_{2}$ is the pomeron coupling to the projectile.

In the alternative realization of RFT only a single pomeron coupling is possible to an elementary (thus not evolved) external source. This assumption naturally emerges if one considers dipole-dipole scattering in QCD. Then, the initial condition of the Hamiltonian evolution is given by:

$$
\tilde{\Psi}(y=0, q)=\Phi_{1}(0) q=g_{1} q
$$

and the scattering amplitude may be obtained as

$$
\tilde{T}\left(Y ; g_{1}, g_{2}\right)=g_{2} \Phi_{1}(Y)=\left.g_{2} \frac{\partial \Psi(Y, q)}{\partial q}\right|_{q=0} .
$$

\subsection{High rapidity asymptotics of the minimal RFT}

The solution to the Hamiltonian problem (2.18) may be given in terms of an infinite summation over a discrete set of eigenstates with wave function $\psi_{n}(q)$

$$
\Psi(y, q)=\sum_{n=0}^{\infty} \lambda_{n} e^{-E_{n} y} \psi_{n}(q)
$$

with the eigenvalues of the Hamiltonian $H$ denoted by $E_{n}, n=0,1,2, \ldots$. Clearly, the asymptotic rapidity dependence of the scattering amplitude is governed by the lowest eigenvalue $E_{0}$.

Minimal RFT is known to exhibit a counter-intuitive feature of an asymptotic decrease of the scattering amplitude down to zero at $Y \rightarrow \infty$. This happens because the lowest eigenvalue $E_{0}$ of the RFT Hamiltonian is positive. We shall recall a heuristic derivation of $E_{0}$, given in Ref. 39] that demonstrates this explicitely.

First, let us transform the eigenfunction corresponding to $E_{0}$ :

$$
\psi_{0}(q)=e^{(q-\varrho)^{2} / 4} f_{0}(q)
$$

that leads to the following equation for $f_{0}(q)$ :

$$
H^{\prime} f_{0}(q)=\left(-\frac{\partial^{2}}{\partial q^{2}}-\frac{1}{2}+\frac{1}{4}(q-\varrho)^{2}\right) f_{0}(q)=\frac{E_{0}}{\lambda q} f_{0}(q)
$$

and the boundary conditions in Eq. (2.19) impose the following behavior of $f_{0}(q)$ :

$$
\begin{aligned}
& f_{0}(q=0)=0 \\
& f_{0}(q \rightarrow \infty) \propto e^{-q^{2} / 4+q \varrho / 2}
\end{aligned}
$$


This eigenvalue problem may be solved approximately starting from a simpler problem of the ground state of the harmonic oscillator, with only modification due the initial condition Eq. (2.31). In this case, the Coulomb term $E_{0} / \lambda q$ is treated as a small perturbation. Then the value $E_{0}$ must be adjusted so that the lowest eigenvalue of the operator $H^{\prime}-E_{0} / \lambda q$ vanishes. Fortunately, the eigenfunctions of operator $H^{\prime}$ in Eq. (2.30) defined on $L_{R}^{2}(0, \infty)$ are known. These are parabolic cylinder functions $D_{\nu}(q-\varrho):$

$$
H^{\prime} D_{\nu}(q-\varrho)=\nu D_{\nu}(q-\varrho) .
$$

It emerges self-consistently that $\nu$ quickly vanishes with increasing $\varrho$, specifically $\nu \sim e^{-\varrho^{2} / 2}$. The initial condition at $q \rightarrow 0$ implies

$$
D_{\nu}(-\varrho)=0,
$$

fulfilled by $\nu=\nu_{0}$ that may be obtained approximately after performing asymptotic expansion of $D_{\nu}(-\varrho)$ in terms of the large parameter $\varrho$ :

$$
\nu_{0} \simeq \frac{\varrho}{\sqrt{2 \pi}} e^{-\varrho^{2} / 2}\left(1+O\left(1 / \varrho^{2}\right)\right) .
$$

In the first order of the perturbative analysis in $E_{0}$, the contribution of the Coulomb term is given by

$$
\left\langle\frac{E_{0}}{\lambda q}\right\rangle \simeq \frac{E_{0}}{\lambda} \int \frac{D_{\nu_{0}}^{2}(q-\varrho)}{q} d q \simeq \frac{E_{0}}{\lambda \varrho}\left(1+O\left(1 / \varrho^{2}\right)\right) .
$$

This correction must be adjusted so that it cancels to zero the energy of the operator $H^{\prime}-E_{0} / \lambda q$. Thus it follows from Eq. (2.33) and Eq. (2.36) that

$$
\frac{E_{0}}{\mu}=\nu_{0}
$$

leading to the energy of the ground state

$$
E_{0} \simeq \frac{\mu \varrho}{\sqrt{2 \pi}} e^{-\varrho^{2} / 2}\left(1+O\left(1 / \varrho^{2}\right)\right)
$$

and the behavior of the RFT wave function at large rapidities,

$$
\Psi(y, q) \sim e^{(q-\varrho)^{2} / 4} D_{\nu_{0}}(q-\varrho) e^{-\frac{y}{\sqrt{2 \pi}} \mu \varrho e^{-\varrho^{2} / 2}} .
$$

Therefore, the scattering amplitudes at fixed external couplings decrease exponentially with increasing rapidity

$$
T\left(Y, g_{1}, g_{2}\right) \sim \exp \left[-\frac{Y}{\sqrt{2 \pi}} \mu \varrho e^{-\varrho^{2} / 2}\right] .
$$

Note, that the exponent $E_{0}$ is exponentially small at large $\varrho, E_{0} \sim e^{-\varrho^{2}}$, and that the splitting of $E_{0}$ from zero is a non-perturbative effect in the triple pomeron coupling, $\lambda=\mu / \varrho$. This exponential decrease of the amplitude may be interpreted as a result of a tunneling phenomenon [36, 39, 40]. In fact, the tunneling rate was explicitly calculated using the instanton calculus [40] which gives exactly the $Y$-dependence given by Eq. (2.40). Thus, the semi-classical calculation of the (eikonal) MRFT scattering amplitude at large $Y$ yields:

$$
T\left(Y, g_{1}, g_{2}\right) \simeq\left(1-e^{-\varrho g_{1}}\right)\left(1-e^{-\varrho g_{2}}\right) \exp \left[-\frac{Y}{\sqrt{2 \pi}} \mu \varrho e^{-\varrho^{2} / 2}\right]
$$




\subsection{Markovian RFT}

Another special case of RFT is obtained for the choice

$$
\alpha^{\prime}=\alpha, \quad \beta^{\prime}=\beta=\alpha^{3},
$$

in the Langevin equation. In this case the evolution of the system may be represented in terms of a Markovian reaction-diffusion process in 0 -dimensions with the $1 \rightarrow 2$ particle splitting probability $\alpha$ and $2 \rightarrow 1$ particle merging probability $\beta=\alpha^{3}$. To be precise, in this case hierarchy of evolution equations (2.10) for moments $\left\langle u^{k}\right\rangle$,

$$
\frac{d\left\langle u^{k}\right\rangle_{y}}{d y}=\alpha k\left\langle u^{k}\right\rangle_{y}+\alpha k(k-1)\left\langle u^{k-1}\right\rangle_{y}-\alpha^{3} k\left\langle u^{k+1}\right\rangle_{y}-\alpha^{3} k(k-1)\left\langle u^{k}\right\rangle_{y},
$$

is identical to the hierarchy of evolution equations (3.11) for factorial moments $n^{(k)}$ of particle number in the reaction-diffusion process. This version of RFT, which we denote as RD-RFT, is defined by the following hierarchy of evolution equations for $k$-pomeron amplitudes

$$
\frac{d \Phi_{k}}{d y}=\mu k \Phi_{k}+\lambda k(k+1) \Phi_{k+1}-\lambda(k-1) \Phi_{k-1}-\lambda^{\prime} k(k+1) \Phi_{k}
$$

with the pomeron intercept $\mu=\alpha$, the triple pomeron coupling $\lambda=\alpha^{2}$ and the quartic $2 \rightarrow 2$ pomeron coupling $\lambda^{\prime}=\alpha^{3}$. Consequently, the Hamiltonian of the system reads

$$
H_{M}=\left(\lambda q^{2}-\mu q\right) \frac{\partial}{\partial q}+\left(\lambda^{\prime} q^{2}-\lambda q\right) \frac{\partial^{2}}{\partial q^{2}} .
$$

In general, the Hamiltonian $H_{M}$ factorizes into a $q$-dependent part (without derivatives) and a $\partial / \partial q$ dependent part provided that $\lambda^{\prime} / \mu=(\lambda / \mu)^{2}$. This condition is obeyed in particular by values of $\lambda$ and $\lambda^{\prime}$ imposed by the relation to the reaction-diffusion model and the Hamiltonian reads

$$
H_{M}=\alpha\left(\alpha q^{2}-q\right)\left(\frac{\partial}{\partial q}+\alpha \frac{\partial^{2}}{\partial q^{2}}\right) .
$$

In order to find the scattering amplitude we consider the quantum mechanical problem defined by this Hamiltonian:

$$
-\frac{\partial \Psi}{\partial y}=H_{M} \Psi
$$

The boundary conditions that should be imposed on $\Psi(y, q)$ in the regular singular points $q=0$ and $q=\varrho=1 / \alpha$ are the following:

$$
\begin{aligned}
& \Psi(y, q=0)=0 \\
& \Psi(y, q) \text { is an analytic function around } q=1 / \alpha .
\end{aligned}
$$

The solution may be represented in terms of the eigenfunctions $\psi_{n}(q)$ and eigenvalues of the Hamiltonian $H_{M}$

$$
\Psi(y, q)=\sum_{n=0}^{n=\infty} \lambda_{n} e^{-E_{n} y} \psi_{n}(q) .
$$


Clearly, the state characterized by

$$
\begin{aligned}
& E_{0}=0 \\
& \psi_{0}(q)=1-e^{-q / \alpha},
\end{aligned}
$$

is an eigenstate. In fact, this is the ground state of $H_{M}$ which dominates the scattering amplitude at high rapidities $y$. All the higher states with $n=1,2, \ldots$ obey the condition ${ }^{2} \psi_{n}(\varrho)=0$, see e.g. Ref. [44]. Therefore, the weight $\lambda_{0}$ in Eq. (2.50) may be found from the initial condition at $y=0$,

$$
\Psi(y=0, q=1 / \alpha)=\lambda_{0} \psi_{0}(1 / \alpha)=\lambda_{0}\left(1-e^{-1 / \alpha^{2}}\right),
$$

and the solution at large $y$ is approximately given by

$$
\Psi(y, q) \simeq \frac{\Psi(0,1 / \alpha)}{1-e^{-1 / \alpha^{2}}}\left(1-e^{-q / \alpha}\right) .
$$

Assuming the eikonal couplings to the external sources, characterized by coupling constants $g_{1}$ and $g_{2}$, the initial condition takes the form $\Psi(0, q)=1-\exp \left(-g_{1} q\right)$, and the scattering amplitude at high $Y$ reads

$$
T\left(Y, g_{1}, g_{2}\right) \simeq \frac{\left(1-e^{-g_{1} / \alpha}\right)\left(1-e^{-g_{2} / \alpha}\right)}{1-e^{-1 / \alpha^{2}}} .
$$

For $g_{1}=g_{2}=\alpha \ll 1$ and $Y \rightarrow \infty$ this amplitude tends to $(1-1 / e)^{2} /\left(1-e^{-1 / \alpha^{2}}\right)$ which is far from the unitarity limit.

In the standard reaction-diffusion picture only coupling of single pomeron to the elementary external source is allowed, with the strength $\alpha$, and $g_{1}=g_{2}=\alpha$. Then, with the initial condition $\Psi(0, q)=q / \alpha$, one obtains for $\alpha Y \gg 1$

$$
\left.\left.\tilde{T}\left(Y, g_{1}, g_{2}\right)\right|_{g_{1}=g_{2}=\alpha} \simeq \frac{\left(g_{1} / \alpha\right)\left(g_{2} / \alpha\right)}{1-e^{-1 / \alpha^{2}}}\right|_{g_{1}=g_{2}=\alpha}=\frac{1}{1-e^{-1 / \alpha^{2}}}
$$

As we will see in the next section, the results for the amplitude given by Eq. (2.55) or Eq. (2.56) may be reproduced in the reaction-diffusion model confirming the equivalence of the approaches.

\section{High energy behavior of the scattering amplitude in diffusion reaction framework}

In this section we use the reaction-diffusion formalism to reproduce the above results in Reggeon field theory. This yields two benefits. First the results of Reggeon field theory emerge rather easily using the reaction-diffusion formalism and, secondly, some seemingly surprising results from the Reggeon field theory point of view have a simple interpretation from the reaction-diffusion point of view.

\footnotetext{
${ }^{2}$ This may be easily seen from an analysis of the power-series solutions of the eigenvalue equation, $H_{M} \psi_{n}=E_{n} \psi_{n}$, around the regular singular point $q=\varrho$. Namely, for any $E_{n} \neq 0$ there exists an analytic solution which approaches zero as $q-\varrho$ at $q \rightarrow \varrho$. The other independent solution does not vanish at $q=\varrho$ but it contains non-analytic terms $\sim \log (\varrho-q)$ and thus it is rejected.
} 


\section{$3.13 P$ coupling only}

High energy scattering where only a three pomeron interaction is present in the Reggeon field theory is a very natural theory to study from the $t$-channel (Reggeon field theory) perspective. It does not naturally correspond to a reaction-diffusion theory but, formally, one can force it into such a description. As we have seen in the previous section the hierarchy equation for the factorial moments, $n^{(k)}$, is

$$
\frac{d n^{(k)}}{d y}=\alpha k n^{(k)}+\alpha k(k-1) n^{(k-1)}-\beta k n^{(k-1)}
$$

with

$$
n^{(k)}(y)=\sum_{n=k}^{\infty} P_{n}(y) n(n-1) \cdots(n-k+1)
$$

and where $P_{n}$ is the probability of having exactly $n$ particles in the system at time (rapidity) $y$. The hierarchy equation is equivalent to the master equation

$$
\frac{d P_{n}}{d y}=-\alpha n P_{n}+\alpha(n-1) P_{n-1}+\beta(n+1)(n+2) P_{n+2}-\beta n(n+1) P_{n+1}
$$

which requires an elementary $1 \rightarrow 2$ splitting with transition rate $\alpha$, a $2 \rightarrow 0$ vertex having transition rate $\beta$ and a $2 \rightarrow 1$ vertex with transition rate $-\beta$. These later two vertices are certainly not natural in a genuine reaction-diffusion process, especially the $2 \rightarrow 1$ vertex with negative probability. Nevertheless, formally, such vertices in a reaction-diffusion context are equivalent to a Reggeon field theory having only a triple pomeron coupling. The presence of a $2 \rightarrow 1$ vertex with a negative transition rate means that $P_{n}$ is not guaranteed to be positive but, as we shall see, this does not appear to cause difficulties in the situation we shall be considering.

The presence of a $2 \rightarrow 0$ vertex means that

$$
\frac{d P_{0}}{d y}=2 \beta P_{2}
$$

so that there are transitions into the zero particle state. However, there are no transitions out of the zero particle state, as is apparent from (3.3), so that ultimately the system will settle down completely into the zero particle sector. However, in the situation of interest to us, $\beta=\alpha^{3}$ is very small so that $P_{2}$ at early times is very small and thus the lifetime of the system is very long, of size $e^{1 / 2 \alpha^{2}}$ as we shall find below. Thus the system will first settle down, rather quickly, into a quasi-stable state and then slowly all the probability will appear in the $n=0$ sector. This slow transition to the $n=0$ state will correspond to the tunneling of the $3 P$ Reggeon field theory discovered long ago. 
To be specific suppose at $y=0$ we take

$$
P_{n}(0)=\delta_{n 1},
$$

that is we start with a single particle. So long as $\alpha / \beta \gg 1$ probability rapidly flows to higher $n$ states, according to (3.3), via $1 \rightarrow 2$ transitions. When $n$ reaches size $\alpha / \beta$ the $2 \rightarrow 0$ and $2 \rightarrow 1$ transitions become important and the system becomes metastable. It is easy to check that

$$
P_{n}=\frac{c}{2}\left(\frac{\alpha}{\beta}\right)^{\frac{n+1}{2}} \frac{(n-2) ! !}{n !} e^{-\alpha /(2 \beta)}
$$

with $(-1) ! ! \equiv 1$, gives $\frac{d P_{n}}{d y}=0$ for $n>1$ when (3.6) is substituted into (3.3) and furthermore that this is the case even when a different value of $c$ is taken for even values of $n$ as compared to odd values of $n$. The $n=0$ part of (3.3) is given by (3.4) which, along with the $n=1$ part of (3.3), is the only departure from (3.6) being a fixed point solution. Now starting from (3.5) we expect that $P_{n}$ should be smooth in $n$ at large values of $n$. In order to insure this smoothness one must choose the $c$ in (3.6) to be different for even and odd values of $n$. A simple calculation shows that if one takes

$$
\begin{array}{cl}
c=1 & \text { for odd } n, \\
c=\sqrt{2 / \pi} & \text { for even } n
\end{array}
$$

then (3.6) is smooth in $n$, for large $n$, and $P_{n}$ is a normalized probability distribution when $\alpha / \beta \gg 1$. One can expect the form (3.6) to set in at a "time"

$$
y_{0} \simeq 1 / 2 \beta
$$

after the start of the evolution of the system from the initial distribution (3.5).

Now as time goes by more and more of the probability of the system will be located in the $n=0$ state. This means that one must change the normalization of (3.6) to account for the loss of probability in the normal $n \neq 0$ states. This is easily done by including a factor $1-P_{0}(y)$ on the right hand side of (3.6). One can then, in general, write (3.4) as

$$
\frac{d}{d y}\left(1-P_{0}\right)=-2 \beta P_{2}\left(1-P_{0}\right)
$$

where $P_{2}$ is given exactly by (3.6). This equation is easily solved as

$$
1-P_{0}(y)=\exp \left[-\frac{y}{\sqrt{2 \pi}}\left(\frac{\alpha^{3}}{\beta}\right)^{1 / 2} e^{-\alpha / 2 \beta}\right]
$$


This is exactly the tunneling probability found by Alessandrini et al. 36, 37, 38, 39, 40, and given in (2.41) above.

The factor multiplying $-y$ in the exponent in (3.10) is to be identified with $\alpha_{P}-1$ with $\alpha_{P}$ the pomeron intercept. The high energy scattering amplitude will decrease at large $y$ a result here which is easily interpreted as the loss of probability from the active states, $n \neq 0$, to the inactive, $n=0$, state. In the reaction diffusion picture there is no tunneling, only a very slow loss of probability from the metastable fixed point configuration of probabilities given by (3.6) into the $n=0$ state.

\section{$3.2 \quad 3 P$ and $4 P$ couplings}

The hierarchy equation (see (2.43) $)$

$$
\frac{d n^{(k)}}{d y}=\alpha k n^{(k)}+\alpha k(k-1) n^{(k-1)}-\beta k n^{(k+1)}-\beta k(k-1) n^{(k)}
$$

along with the corresponding master equation

$$
\frac{d P_{n}}{d y}=-\alpha n P_{n}+\alpha(n-1) P_{n-1}+\beta n(n+1) P_{n+1}-\beta n(n-1) P_{n}
$$

correspond to a genuine reaction diffusion process with a $1 \rightarrow 2$ vertex of strength $\alpha$ and a $2 \rightarrow 1$ vertex of strength $\beta$, see e.g. Ref. [29]. In Reggeon field theory language the additional term in (3.11), as compared to (3.1), corresponds to a $4 P$ coupling. The fixed point solution to (3.11) and (3.12) is

$$
P_{n}=\frac{1}{n !}\left(\frac{\alpha}{\beta}\right)^{n} \frac{1}{e^{\alpha / \beta}-1}
$$

and

$$
n^{(k)}=(\alpha / \beta)^{n} \frac{e^{\alpha / \beta}}{e^{\alpha / \beta}-1} .
$$

Eq. (3.12) does not have transitions to the $n=0$ state so if one takes a normalized initial condition having $P_{0}=0$ then $P_{0}(y)$ will remain equal to zero. When $\alpha / \beta \gg 1$ the fixed point solution is very close to a Poisson distribution of probabilities. The fact that there is now a genuine fixed point solution to the reaction-diffusion problem guarantees that $\alpha_{P}=1$ in the solution to the Reggeon field theory, and this indeed is the case as we have seen in Sec. 2.5. 


\subsection{High energy amplitudes}

As discussed earlier in Sec. 2.3 it is not so straightforward to decide how to define a high energy scattering amplitude. In Reggeon field theories one has to decide how the pomeron field, the dynamical variable in the theory, couples to external particles which are not part of the dynamics of the theory. The most common choice, given in (2.24) and (2.25), has been to directly couple the pomeron field to a source at $y=0$ with strength, say, $g_{1}$ and to another source of strength, say, $g_{2}$ at $y=Y$. In the following we shall deal exclusively with the model described in Sec. 3.2 as this seems the more interesting case. The $3 P$ case is easily treated by the same methods and the result is as in (2.41).

Once an $s$-channel picture of the dynamics is introduced more constraints on the definition of the scattering emerge. In the first place the particles which scatter are the same as the particles making up the $s$-channel dynamics. Secondly, the scattering is no longer manifestly boost invariant. For example, in the center of mass the scattering looks like a scattering of two highly evolved systems while in the rest frame of one of the particles the scattering looks like that of a highly evolved system on an elementary quantum. For both (3.1) and (3.11) boost invariance can be satisfied by taking the scattering amplitude to be

$$
T(Y)=\sum_{k=1}^{\infty} \frac{(-1)^{k-1}}{k !} \alpha^{2 k} n^{(k)}\left(Y_{0}\right) n^{(k)}\left(Y-Y_{0}\right)
$$

where $Y_{0}$ is arbitrary. Eq. (3.15) represents scattering as the sum over $k$ interactions of elementary quanta in one, say left-moving, system evolved to rapidity $Y-Y_{0}$ and another, right-moving, system evolved to rapidity $Y_{0}$. The elementary interactions are of strength $\alpha^{2}$ and $T$ is independent of $Y_{0}$ if $\alpha^{3}=\beta$. The use of factorial moments seems necessary in (3.15) in order to get boost invariance. At large $y$ values $n^{(k)}(y)$ approaches the fixed point solution given in (3.14), independent of the initial condition, in the case of a genuine reaction diffusion process corresponding to $3 P$ and $4 P$ interactions. $T$ is most easily evaluated by taking $Y_{0}=0$ in which case

$$
T(Y)=\alpha^{2} n^{(1)}(Y)
$$

which has the large $Y$ limit

$$
T(Y) \underset{Y \rightarrow \infty}{\sim} \alpha^{3} / \beta \frac{1}{1-e^{\alpha / \beta}} \simeq 1+e^{-1 / \alpha^{2}}
$$

where we have taken $\alpha^{3}=\beta$ in the right-most expression in (3.17). This agrees with the result found from Reggeon field theory in (2.56). The fact that $T$ is very near one at large $Y$ and small $\alpha$ follows from the fixed point properties of the theory along with boost invariance and is independent of the initial condition for the system so long as the initial states are normalized to unit probability. 
Now let us evaluate the scattering amplitude in the eikonal model, repeating the derivation given in Sec. 2.5 but from a different perspective. It is still convenient to use reaction-diffusion dynamics, but we shall define $T$ in the way that has been traditional in Reggeon field theories. That is, we take, corresponding to (2.25),

$$
T(Y)=\sum_{k=1}^{\infty} \frac{(-1)^{k-1}}{k !}\left(g_{2} \alpha\right)^{k} n^{(k)}(Y)
$$

where $n^{(k)}$ satisfies the hierarchy equations (3.11) of the Reggeon field theory. The initial condition for $n^{(k)}$ is

$$
n^{(k)}(0)=\left(g_{1} / \alpha\right)^{k}
$$

expressing the eikonal model of pomeron interactions with the external particle (source) at $y=0$. Now (3.19) requires

$$
P_{n}(0)=\frac{1}{n !}\left(g_{1} / \alpha\right)^{n} e^{-g_{1} / \alpha}
$$

We note that

$$
\sum_{n=1}^{\infty} P_{n}(0)=1-e^{-g_{1} / \alpha}
$$

so that the initial state is not properly normalized from the $s$-channel (reaction-diffusion) point of view. Eq. (3.21) gives

$$
n^{(k)}(Y) \underset{Y \rightarrow \infty}{\sim}\left(1 / \alpha^{2}\right)^{k}\left(1-e^{-g_{1} / \alpha}\right) \frac{1}{1-e^{-1 / \alpha^{2}}}
$$

which, when used in (3.18), gives, as in (2.55),

$$
T(Y) \underset{Y \rightarrow \infty}{\widetilde{1}}\left(1-e^{-g_{2} / \alpha}\right)\left(1-e^{-g_{1} / \alpha}\right) \frac{1}{1-e^{-1 / \alpha^{2}}}
$$

or

$$
T(Y) \underset{Y \rightarrow \infty}{\widetilde{1}}\left(1-e^{-g_{2} / \alpha}\right)\left(1-e^{-g_{1} / \alpha}\right)
$$

where we have dropped small terms, of size $e^{-1 / \alpha^{2}}$, in obtaining (3.24) which is the same as given in Refs. [38, 39, 13].

Eq. (3.24) expresses "gray" rather than "black" scattering. From the Reggeon field theory point of view this result is rather mysterious; why should a strongly interacting scattering not saturate unitarity at high energy? From the reaction-diffusion ( $s$-channel) picture the factors in (3.24) just represent the normalization of the states (see (3.21) ) involved in the scattering. 


\subsection{Generating functions}

For completeness, let us finish with a brief discussion of a generating function, $Z(y, v)$, for occupation number probabilities, $P_{n}(y)$, in the reaction diffusion process,

$$
Z(y, v)=\sum_{n=1}^{\infty} P_{n}(y) v^{n}
$$

where $v$ is an auxiliary variable. The probabilities of $n$-particle states may be expressed as

$$
P_{n}(y)=\left.\frac{1}{n !} \frac{\partial^{n} Z(y, v)}{\partial v^{n}}\right|_{v=0},
$$

and the factorial moments read

$$
n^{(k)}(y)=\left.\frac{\partial^{k} Z(y, v)}{\partial v^{k}}\right|_{v=1} .
$$

The generating function is intimately connected to the wave function of the corresponding RFT, given by (2.20). Using (2.11), (2.20), the identification $\left\langle u^{k}\right\rangle_{y}=n^{(k)}(y)$ and the relation in (3.27) we obtain

$$
\Psi(y, q)=-\left.\sum_{k=1}^{\infty} \frac{1}{k !} \frac{\partial^{k} Z(y, v)}{\partial v^{k}}\right|_{v=1}(-\alpha q)^{k},
$$

which may be simplified to

$$
\Psi(y, q)=Z(y, 1)-Z(y, 1-\alpha q) .
$$

It is straightforward to verify that the inverse relation reads

$$
Z(y, v)=\Psi(y, 1 / \alpha)-\Psi(y,(1-v) / \alpha)
$$

\section{Acknowledgments}

We are grateful to J. Bartels, C. Ewerz, E. Iancu, S. Munier, M. Salvadore and G.P. Vacca for enlightening discussions. A.M. and A. Sh. wish to thank the DESY theory group for their hospitality during their visit when this work was being initialized. S.B. thanks the Minerva foundation for its support, L.M. gratefully acknowledges the support of the grant of the Polish State Committee for Scientific Research No. 1 P03B 028 28. A.M. and B.X. are partially supported by the US Department of Energy. A. Sh. acknowledges financial support by the Deutsche Forschungsgemeinschaft under contract Sh $92 / 2-1$.

\section{References}

[1] L. N. Lipatov, "Reggeization Of The Vector Meson And The Vacuum Singularity In Nonabelian Gauge Theories," Sov. J. Nucl. Phys. 23 (1976) 338 [Yad. Fiz. 23 (1976) 642]. 
[2] E. A. Kuraev, L. N. Lipatov and V. S. Fadin, "The Pomeranchuk Singularity In Nonabelian Gauge Theories," Sov. Phys. JETP 45 (1977) 199 [Zh. Eksp. Teor. Fiz. 72 (1977) 377].

[3] I. I. Balitsky and L. N. Lipatov, "The Pomeranchuk Singularity In Quantum Chromodynamics," Sov. J. Nucl. Phys. 28 (1978) 822 [Yad. Fiz. 28 (1978) 1597].

[4] L. N. Lipatov, "Small-x physics in perturbative QCD," Phys. Rept. 286 (1997) 131.

[5] L. V. Gribov, E. M. Levin and M. G. Ryskin, "Semihard Processes In QCD," Phys. Rept. 100 (1983) 1.

[6] J. Bartels, "Unitarity corrections to the Lipatov pomeron and the four gluon operator in Z. Phys. C 60 (1993) 471.

[7] J. Bartels and M. Wüsthoff, "The Triple Regge limit of diffractive dissociation in deep inelastic Z. Phys. C 66 (1995) 157.

[8] J. Bartels and C. Ewerz, "Unitarity corrections in high-energy QCD," JHEP 9909 (1999) 026.

[9] C. Ewerz, "Reggeization in high energy QCD," JHEP 0104 (2001) 031.

[10] M. A. Braun, "Nucleus nucleus scattering in perturbative QCD with N(c) $\rightarrow$ infinity," Phys. Lett. B 483 (2000) 115.

[11] M. A. Braun, "Nucleus nucleus interaction in the perturbative QCD," Eur. Phys. J. C 33 (2004) 113.

[12] M. A. Braun, "Conformal invariant pomeron interaction in the perurbative QCD with large N(c)," Phys. Lett. B 632 (2006) 297.

[13] S. Bondarenko and L. Motyka, "Solving effective field theory of interacting QCD pomerons in the semi-classical approximation," arXiv:hep-ph/0605185.

[14] A. H. Mueller, "Soft Gluons In The Infinite Momentum Wave Function And The Bfkl Pomeron," Nucl. Phys. B 415 (1994) 373.

[15] A. H. Mueller and B. Patel, "Single And Double Bfkl Pomeron Exchange And A Dipole Picture Of High-Energy Nucl. Phys. B 425 (1994) 471.

[16] A. H. Mueller, "Unitarity and the BFKL pomeron," Nucl. Phys. B 437 (1995) 107.

[17] I. Balitsky, "Operator expansion for high-energy scattering," Nucl. Phys. B 463 (1996) 99.

[18] J. Jalilian-Marian, A. Kovner and H. Weigert, "The Wilson renormalization group for low $\mathrm{x}$ physics: Gluon evolution at finite parton density," Phys. Rev. D 59 (1999) 014015; 
[19] J. Jalilian-Marian, A. Kovner, A. Leonidov and H. Weigert, "The Wilson renormalization group for low x physics: Towards the high density regime," Phys. Rev. D 59 (1999) 014014.

[20] Y. V. Kovchegov, "Small-x F2 structure function of a nucleus including multiple pomeron exchanges," Phys. Rev. D 60 (1999) 034008.

[21] Y. V. Kovchegov, "Unitarization of the BFKL pomeron on a nucleus," Phys. Rev. D 61 (2000) 074018.

[22] E. Iancu, A. Leonidov and L. D. McLerran, "Nonlinear gluon evolution in the color glass condensate. I," Nucl. Phys. A 692 (2001) 583.

[23] E. Iancu, A. Leonidov and L. D. McLerran, "The renormalization group equation for the color glass condensate," Phys. Lett. B 510 (2001) 133.

[24] E. Iancu and L. D. McLerran, "Saturation and universality in QCD at small x," Phys. Lett. B 510 (2001) 145;

[25] E. Ferreiro, E. Iancu, A. Leonidov and L. McLerran, "Nonlinear gluon evolution in the color glass condensate. II," Nucl. Phys. A 703 (2002) 489.

[26] A. H. Mueller and A. I. Shoshi, "Small-x physics beyond the Kovchegov equation," Nucl. Phys. B 692 (2004) 175.

[27] E. Iancu, A. H. Mueller and S. Munier, "Universal behavior of QCD amplitudes at high energy from general tools of statistical physics," Phys. Lett. B 606 (2005) 342.

[28] E. Levin and M. Lublinsky, "Balitsky's hierarchy from Mueller's dipole model and more about target Phys. Lett. B 607 (2005) 131.

[29] E. Iancu and D. N. Triantafyllopoulos, "A Langevin equation for high energy evolution with pomeron loops," Nucl. Phys. A 756 (2005) 419.

[30] A. H. Mueller, A. I. Shoshi and S. M. H. Wong, "Extension of the JIMWLK equation in the low gluon density region," Nucl. Phys. B 715 (2005) 440.

[31] E. Levin and M. Lublinsky, "Towards a symmetric approach to high energy evolution: Generating functional with Pomeron loops," Nucl. Phys. A 763 (2005) 172.

[32] E. Iancu and D. N. Triantafyllopoulos, "Non-linear QCD evolution with improved triple-pomeron vertices," Phys. Lett. B 610 (2005) 253.

[33] E. Iancu, G. Soyez and D. N. Triantafyllopoulos, "On the probabilistic interpretation of the evolution equations with Pomeron loops in QCD," Nucl. Phys. A 768 (2006) 194. 
[34] D. N. Triantafyllopoulos, "Pomeron loops in high energy QCD," Acta Phys. Polon. B 36 (2005) 3593.

[35] D. Amati, L. Caneschi and R. Jengo, "Summing Pomeron Trees," Nucl. Phys. B 101 (1975) 397.

[36] V. Alessandrini, D. Amati and R. Jengo, Nucl. Phys. B 108 (1976) 425.

[37] R. Jengo, "Zero Slope Limit Of The Pomeron Field Theory," Nucl. Phys. B 108 (1976) 447.

[38] D. Amati, M. Le Bellac, G. Marchesini and M. Ciafaloni, Nucl. Phys. B 112 (1976) 107.

[39] M. Ciafaloni, M. Le Bellac and G. C. Rossi, "Reggeon Quantum Mechanics: A Critical Discussion," Nucl. Phys. B 130 (1977) 388.

[40] M. Ciafaloni, "Instanton Contributions In Reggeon Quantum Mechanics," Nucl. Phys. B 146 (1978) 427.

[41] P. Rembiesa and A. M. Staśto, "Algebraic models for the hierarchy structure of evolution equations at small x," Nucl. Phys. B 725 (2005) 251.

[42] A. Kovner and M. Lublinsky, "More remarks on high energy evolution," Nucl. Phys. A 767 (2006) 171.

[43] A. I. Shoshi and B. W. Xiao, "Pomeron loops in zero transverse dimensions," Phys. Rev. D 73 (2006) 094014.

[44] M. Kozlov and E. Levin, "Solution for the BFKL pomeron calculus in zero transverse dimensions," arXiv:hep-ph/0604039.

[45] A. I. Shoshi and B. W. Xiao, "Diffractive dissociation including pomeron loops in zero transverse dimensions," arXiv:hep-ph/0605282.

[46] J. P. Blaizot, E. Iancu and D. N. Triantafyllopoulos, "A zero-dimensional model for high-energy scattering in QCD," arXiv:hep-ph/0606253. 